Published in final edited form as:

Int J Tuberc Lung Dis. 2014 June ; 18(6): 682-688. doi:10.5588/ijtld.13.0729.

\title{
Role of oral candidiasis in TB and HIV co-infection: AIDS Clinical Trial Group Protocol A5253
}

\section{H. Shiboski ${ }^{\star}$, H. Chen ${ }^{\dagger}$, M. A. Ghannoum ${ }^{\ddagger}$, L. Komarow§, S. Evans ${ }^{\S}$, P. K. Mukherjee ${ }^{\ddagger}$, N. Isham ${ }^{\ddagger}$, D. Katzenstein ", A. Asmelash ${ }^{\#}$, A. E. Omozoarhe ${ }^{\star \star}$, S. Gengiah ${ }^{\dagger \dagger}$, R. Allen ${ }^{\ddagger \ddagger}$, S.

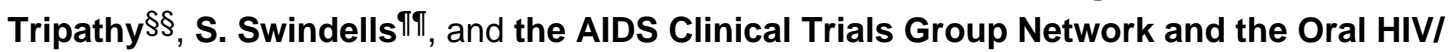 AIDS Research Alliance}

*Department of Orofacial Sciences, School of Dentistry, University of California San Francisco, San Francisco, California

†Center for Biostatistics in AIDS Research, Harvard School of Public Health, Boston, Massachusetts

¥Center for Medical Mycology, Department of Dermatology, University Hospitals Case Medical Center and Case Western Reserve University, Cleveland, Ohio

$\S$ Statistical Data Analysis Center, Harvard School of Public Health, Boston, Massachusetts

IStanford University Medical Center, Stanford, California, USA

\#Scottish Livingstone Hospital, Molepole

${ }^{* *}$ Princess Marina Hospital, Gaborone, Botswana

${ }^{+\dagger}$ Centre for the AIDS Programme of Research in South Africa, Durban, South Africa

¥¥AIDS Clinical Trial Group Operations Center, Silver Spring, Maryland, USA

$\S \S$ Molecular Virology Clinic National AIDS Research Institute, Maharashtra Industrial Development Corporation, Bhosari, India

IT/Internal Medicine/Infectious Diseases University of Nebraska Medical Center, Omaha, Nebraska, USA

\section{SUMMARY}

OBJECTIVE-To evaluate the association between oral candidiasis and tuberculosis (TB) in human immunodeficiency virus (HIV) infected individuals in sub-Saharan Africa, and to investigate oral candidiasis as a potential tool for TB case finding.

METHODS-Protocol A5253 was a cross-sectional study designed to improve the diagnosis of pulmonary TB in HIV-infected adults in high TB prevalence countries. Participants received an

\footnotetext{
(C) 2014 The Union

Correspondence to: Caroline H Shiboski, Department of Orofacial Sciences, Box 0422, Room S612, 513 Parnassus Avenue, University of California San Francisco, San Francisco, CA 94143-0422, USA. Tel: (+1) 415476 5976. Fax: (+1) 4154764204. caroline.shiboski@ucsf.edu.

Conflict of interest: none declared.
} 
oral examination to detect oral candidiasis. We estimated the association between TB disease and oral candidiasis using logistic regression, and sensitivity, specificity and predictive values.

RESULTS-Of 454 participants with TB culture results enrolled in African sites, the median age was 33 years, $71 \%$ were female and the median CD4 count was 257 cells $/ \mathrm{mm}^{3}$. Fifty-four (12\%) had TB disease; the prevalence of oral candidiasis was significantly higher among TB cases (35\%) than among non-TB cases $(16 \%, P<0.001)$. The odds of having TB was 2.4 times higher among those with oral candidiasis when controlling for CD4 count and antifungals (95\% CI 1.2-4.7, $P=$ 0.01 ). The sensitivity of oral candidiasis as a predictor of TB was 35\% (95\% CI 22-48) and the specificity $85 \%$ (95\%CI 81-88).

CONCLUSION-We found a strong association between oral candidiasis and TB disease, independent of CD4 count, suggesting that in resource-limited settings, oral candidiasis may provide clinical evidence for increased risk of TB and contribute to TB case finding.

\section{Keywords}

oral candidiasis; tuberculosis; HIV; acquired immunedeficiency syndrome

THE IDENTIFICATION of human immunodeficiency virus (HIV) infected persons with low CD4+cell count is important in areas of high tuberculosis (TB) prevalence, as this group is at highest risk of having TB disease concomitantly with HIV infection. Several studies have shown that oral candidiasis and TB disease were the most common comorbidities among populations with advanced HIV disease: one study among 356 hospitalized Cambodian patients with the acquired immunedeficiency syndrome (AIDS) revealed that oral candidiasis (51\%) and pulmonary and extra-pulmonary TB (44\%) were the most frequent opportunistic infections. ${ }^{1}$ Another study among 205 HIV-infected adults in Malaysia showed that oral candidiasis was the most common mucocutaneous disease, and significant co-existence was found with the major opportunistic systemic diseases such as $\mathrm{TB}^{2}$

Early in the HIV epidemic, oral candidiasis was found to be among the strongest clinical predictors of progression to AIDS in various HIV-infected populations. ${ }^{3,4}$ Oral candidiasis has also been shown to be the most common oral manifestation of HIV infection in different parts of the world, ${ }^{5-14}$ and to be closely associated with low CD4+ cell count. ${ }^{5,7,8,15-20} \mathrm{~A}$ diagnosis of oral candidiasis is usually made by visual examination of the mouth, and nondental health care providers can be readily trained to perform such an examination. ${ }^{19}$ The strong positive association between oral candidiasis and a low CD4+cell count is thus a useful finding with respect to the need for inexpensive surrogate markers of HIV disease progression and related comorbidities in resource-limited countries where measurement of CD4+cell counts is not widely available.

A visual inspection of the soft tissues of the mouth is quick ( $<5 \mathrm{~min}$ ), non-invasive, and inexpensive. It could be used as initial screening to identify patients with oral candidiasis who are most likely to have CD4+cell count $<200$ cells $/ \mathrm{mm}^{3}$, and thus at higher risk for TB disease that may or may not yet be symptomatic. 
The objective of the present study was to evaluate the value of oral candidiasis as a predictor of TB disease among HIV-infected men and women in resource-limited settings with high TB prevalence.

\section{METHODS}

\section{Study design and population}

This study was conducted as part of the AIDS Clinical Trial Group (ACTG) Protocol A5253 in collaboration with the Oral HIV/AIDS Research Alliance (OHARA). ${ }^{21}$ Protocol A5253 was a cross-sectional study designed to construct a standardized diagnostic evaluation (SDE) that improves diagnosis of pulmonary TB as compared to standard-of-care (SOC) TB screening in HIV-infected participants not currently receiving antiretroviral therapy (ART). ${ }^{22}$

The target population for Protocol A5253 was HIV-infected males and females aged $\geq 13$ years living in high TB prevalence regions in sub-Saharan Africa, India and South America. In addition to being HIV-infected, eligibility criteria included not having received ART, or a diagnosis of pulmonary TB, or medications to treat TB within 90 days before study entry. Participants were recruited between 5 February and 30 November 2010 from 11 clinical trials units of the ACTG by clinical site investigators using a convenience sampling strategy. Sites were required to be in a country with a TB incidence of $>60$ per 100000 population per year according to the World Health Organization (WHO) Global Health Atlas country profiles. ${ }^{23}$ The institutional review boards or ethics committees of the participating institutions approved the study, and each participant gave written informed consent. The present report focuses on the African sites only, as $96 \%$ of the TB cases were observed in these sites.

\section{Clinical data collection}

All participants in Protocol A5253 were screened according to SOC and candidate SDE elements. ${ }^{22}$ Sputum culture in either solid or liquid culture was used as the gold standard to define a participant as TB-positive. SOC screening algorithm components were cough, fever, weight loss and/or night sweats occurring within the previous 30 days, Ziehl-Neelsen sputum smear and chest radiography (CXR) for non-pregnant participants. SDE screening included additional symptoms and clinical signs, and sputum smear using fluorescence microscopy. All participants underwent all evaluations. A detailed description of these methods has been published elsewhere. ${ }^{22}$

All participants also underwent a standardized oral soft tissue examination performed by a nurse or a physician who had been trained and calibrated in the diagnosis of common HIVrelated oral mucosal diseases using a protocol developed by the Clinical Science Unit of OHARA. Examiners were trained by watching a 1-h presentation that described how to perform an oral soft tissue examination, and how to diagnose pseudomembranous candidiasis, erythematous candidiasis and angular cheilitis, which are all part of the spectrum of oral candidiasis, as well as other common oral lesions. We used published case definitions of these oral endpoints. ${ }^{24}$ Examiners were considered calibrated if they obtained 
$80 \%$ correct answers to a post-test of clinical slides administered after the training. Participants found to have clinical signs of oral candidiasis had a swab (BBL ${ }^{\mathrm{TM}}$ Culture$\mathrm{Swab}^{\mathrm{TM}}$ kit, BD, Sparks, MD, USA) collected from a well-defined area for culture confirmation of the clinical diagnosis. If a participant had more than one form of oral candidiasis, the examiner was instructed to preferentially collect a swab from a pseudomembranous candidiasis lesion if present. If pseudomembranous candidiasis was not present, but angular cheilitis was observed together with erythematous candidiasis, then the examiner was instructed to collect the swab from the angular cheilitis.

\section{Oral swab specimen processing}

Collection supplies were distributed to clinical trial sites from the OHARA/ACTG Candida repository at the Center for Medical Mycology, Case Western Reserve University, Cleveland, OH, USA. These consisted of culturette swabs containing Amies Transport Media (Becton Dickinson, Franklin Lakes, NJ, USA), dehydrated potato dextrose agar (PDA) (BD) and $4 \mathrm{ml}$ Wheaton vials for preparing agar slants (Wheaton Industries, Millville, NJ, USA). After collection, oral swabs were forwarded to a local laboratory for processing onto PDA (potato dextrose agar) plates for Candida colony count, which were recorded. A subculture of each distinct colony type suspected of being a Candida isolate was then prepared on an agar slant and forwarded to the Case repository for speciation.

\section{Statistical analyses}

Summary statistics were used to describe participant characteristics (sex, age, region of residence and CD4 cell count) by TB disease status. We computed the prevalence of the three types of oral candidiasis and of other oral mucosal lesions, and used the $\chi^{2}$ test (or Fisher's exact test for smaller cell sizes) to explore whether an association existed between these oral outcomes and TB. We fit a stepwise logistic regression model to investigate the association between oral candidiasis and TB disease while controlling for potential confounders. We started by fitting a model in which we included all variables that were suspected confounders of the association between oral candidiasis and TB: CD4 cell count, sex, age and receipt of antifungal medication at the time of study examination. We then performed backward elimination analysis to remove variables that were not associated with TB at a significance level of 0.2. The model yielded adjusted odds ratios (aORs) with $95 \%$ confidence intervals (CIs). We initially also included an interaction term in the logistic regression model to detect whether a possible effect modification existed between CD4 cell count and oral candidiasis, but found no significant interaction effect $(P=0.33)$. To investigate the role of oral candidiasis as a predictor of TB, we estimated the sensitivity, specificity, and positive (PPV) and negative predictive values (NPV) in relation to TB.

\section{RESULTS}

Among 521 participants enrolled in Protocol A5253 in African sites, 455 had an interpretable TB sputum culture result, and 454 underwent an oral examination (and are included in the present report). The majority were Black non-Hispanic (99\%) and female (71\%); 54 had culture-confirmed TB disease (12\%; Table 1); $18 \%$ had a clinical diagnosis of oral candidiasis. The median age was 33 years, and the median CD4+ cell count was 257 
cells $/ \mathrm{mm}^{3}$. As previously reported, participants with and those without TB were similar with regard to age and sex; however, the median CD4 cell count was significantly lower among TB cases (202 cells $/ \mathrm{mm}^{3}$, range 2-699) than in non-cases $\left(270\right.$ cells $/ \mathrm{mm}^{3}$, range $1-1796, P$ $=0.003) .{ }^{22}$ Among participants with $\mathrm{TB}, 2 \%$ were using an antifungal medication at the time of study entry compared to $9 \%$ among those without TB $(P=0.10)$.

The prevalence of oral candidiasis was significantly higher among TB cases (35\%) than among non-cases $(16 \% ; P<0.001)$, predominantly pseudomembranous candidiasis (Table 2). Among 77 cases with a clinical diagnosis of oral candidiasis who had a swab collected for culture (4 cases did not have a confirmatory swab), 62 (81\%) were culture-confirmed. The proportion of confirmation using culture differed by type of candidiasis, with angular cheilitis and pseudomembranous candidiasis having the highest level of culture confirmation (100\% and $85 \%$, respectively), and erythematous candidiasis the lowest (72\%).

Other types of oral lesions had low prevalence ( $\$ 4 \%$ for each type of condition), and included hairy leukoplakia $(n=7)$, recurrent herpes simplex infection $(n=6)$, necrotizing ulcerative gingivitis/periodontitis $(n=6)$, oral Kaposi 's sarcoma $(n=3)$, oral warts $(n=2)$ and recurrent aphthous ulcers $(n=1)$.

Among those with oral candidiasis, $68 \%$ had a CD4 cell count $<200$ cells $/ \mathrm{mm}^{3}$, while among those without oral candidiasis $74 \%$ had a CD4 cell count $>200$ cells $/ \mathrm{mm}^{3}$ (Table 3 ). We found a strong positive association between low CD4 cell count and the presence of oral candidiasis, using a contingency table approach or a non-parametric analysis.

A multivariate logistic regression model exploring the association between TB (outcome) and oral candidiasis revealed that the odds of having TB was 2.4 times higher among those with oral candidiasis when controlling for CD4 count and antifungal medication (95\% CI $1.2-4.7, P=0.01$; Table 4).

The proportion of participants with TB who also had oral candidiasis (sensitivity) was 35\% (95\% CI 22-48). The specificity was 85\% (95\%CI 81-88). Both PPV and NPV of oral candidiasis in relation to TB are presented as functions of TB prevalence in the Figure. For example, for a TB prevalence ranging from $10 \%$ to $15 \%$, the PPV would range from $20 \%$ to $29 \%$, and if TB prevalence was as high at $20 \%$, the PPV of oral candidiasis would be $37 \%$.

\section{DISCUSSION}

This analysis among HIV-infected participants in ACTG Protocol A5253 revealed that oral candidiasis was the most common type of oral mucosal disease. Furthermore, as reported in other studies in sub-Saharan Africa, there was a strong positive association between the presence of oral candidiasis and low CD4 cell count. ${ }^{19}$ Clinical diagnoses of oral candidiasis made by nurses and physicians at various ACTG sites, which were confirmed by a positive culture in more than $80 \%$ of the cases, were strongly associated with TB even after adjusting for CD4 count. The sensitivity was only $35 \%$, while the specificity was $85 \%$. The sensitivity of oral candidiasis was somewhat similar to that of other clinical symptoms described in the primary A5253 paper, such as fever, night sweats and fatigue, which were 43\%, 48\% and $50 \%$, respectively. ${ }^{22}$ The specificity for these symptoms was lower than that of oral 
candidiasis: $71 \%, 61 \%$ and $60 \%$, respectively. Furthermore, while a history of cough in the last 30 days had the highest sensitivity of all clinical symptoms (82\%), specificity was low (39\%). In the A5253 primary paper, performance characteristics were assessed for multiple combinations of clinical symptoms, CD4 cell count, CXR and/or lymphadenopathy. None of these combinations improved upon the predictive values of symptoms of cough, fever, night sweats and fatigue, which are used in TB case finding. The present analysis reveals that due to its strong association with TB disease and high specificity, and despite its modest sensitivity, oral candidiasis should also prompt further evaluation for TB disease among HIV-infected adults.

The strength of the present study is that it specifically explored the association between oral candidiasis and TB by including a systematic oral examination among all participants, regardless of oral symptoms. Furthermore, the clinical diagnosis of oral candidiasis was confirmed by an oral swab culture, and revealed a high proportion of correct clinical diagnoses. Oral examinations were performed by nurses/physicians who had received the same standardized training on the diagnosis of the various forms of oral candidiasis. ${ }^{24}$ The standardized oral examination training module has been evaluated within ACTG clinical research sites (CRS) as part of a recent study to assess the accuracy of oral diagnoses made by CRS examiners (non-dental health care professionals). Among $328 \mathrm{HIV}$-infected participants, the sensitivity and specificity for the diagnosis of oral candidiasis by trained CRS examiners as compared to Oral Medicine specialists as reference standard was $90 \%$ and $92 \%$, respectively, with 153 cases of oral candidiasis diagnosed in this cross-sectional study (unpublished data). The limitations of our study include a potential lack of power, as there were only 54 cases of TB. Use of sputum culture as reference standard excluded cases of extra-pulmonary disease. The proportion of participants currently taking an antifungal medication (8\%) may have slightly diluted the magnitude of the association, although there was no evidence of preferential bias as these participants were evenly distributed by TB status.

The WHO, the Joint United Nations Programme on HIV/AIDS and the Stop TB Partnership have set a target of halving TB mortality rates among HIV-positive people by 2015 compared to $2004 .^{25} \mathrm{WHO}$ recommendations on the interventions needed to prevent, diagnose and treat TB in people living with HIV have been available since $2004{ }^{26}$ The plan recommends that all people living with HIV should be regularly screened for TB using a clinical symptom-based algorithm consisting of current cough, fever, weight loss or night sweats at the time of initial presentation for HIV care and at every visit to a health care facility or contact with a health care worker afterwards. Oral candidiasis is strongly associated with TB disease, and can be assessed by a quick visual examination of the mouth. Like other clinical symptoms, it therefore represents a useful clinical marker to identify a high-risk population who must be screened for TB disease. While many studies have reported on the strong association between oral candidiasis and low CD4 cell count, the present study is among the first to detect a strong association between oral candidiasis and TB disease after controlling for CD4 cell count. It is consistent with findings from a study of 2086 adults with HIV disease in South Africa that revealed that TB and oral candidiasis were the only opportunistic infections to commonly occur among those with CD4 cell counts $\geq 200$ cells $/ \mathrm{mm}^{3.27}$ Among those with CD4 cell count between 201 and 350 
cells $/ \mathrm{mm}^{3}$, the incidence rate of TB and oral candidiasis was 5.7 (95\% CI 3.7-7.8) and 15.2 (95\%CI 11.6-18.8) per 100 person-years, respectively, while all other opportunistic infections had an incidence rate of $<2.5$.

Based on the modest sensitivity and PPV reported by our study, oral candidiasis cannot be used as a diagnostic test for TB disease. However, the strong association found between the presence of oral candidiasis and TB disease independently of CD4 cell count, and the high specificity of oral candidiasis in relation to TB, suggest that it could be used to identify those who should be screened for TB disease, for example with culture and/or nucleic acid amplification tests. Consideration may also be given to use of the urine lipoarabinomannan assay, which has high sensitivity among patients with advanced immunodeficiency. ${ }^{28} \mathrm{In}$ resource-limited countries, it is useful to have a clinical tool that may help identify those at highest risk for TB, and we recommend that TB screening be performed on all HIV-infected patients with a diagnosis of oral candidiasis.

\section{Acknowledgments}

The authors would like to express their sincere appreciation to A5253 study participants, to L-J Deng for her valuable contribution to data analysis, to I Rodriguez-Chavez and J Greenspan for reviewing the manuscript, and to the investigative teams of the ACTG clinical research sites that enrolled participants in Protocol A5253.

The list of sites and contributors to Protocol A5253 is as follows: R R Gangakhedkar and A Risbud (NARI Pune Clinical Research Site [CRS], Pune, India: Site 11601) Clinical Trials Unit (CTU) Grant 1U01 AI069417-01; S Selebaleng and R Molefe (Molepolole Prevention \& Treatment Trials CRS, Molepolole, Botswana: Site 12702) CTU Grant 1U01 AI069456-01; K Kooreng and M Raesi (Gaborone Prevention \& Treatment Trials CRS, Gaborone, Botswana: Site 12701) CTU Grant 1U01 AI069456-01; K Naidoo (CAPRISA eThekwini CRS, Durban, South Africa: Site 31422) CTU Grant 1U01 AI069469-01; S Pillay (Durban International CRS, Durban, South Africa: Site 11201) CTU Grant 1U01 Al069432-01; D Kadam and V Mave (BJ Medical College CRS, Pune, India: Site 31441) CTU Grant 1U01 AI069465-01; P Ive and M Hero (University of the Witwatersrand Helen Joseph CRS, Johannesburg, South Africa: Site 11101) CTU Grant 1U01 AI069463-01; L Mohapi and R Lourens (Soweto AIDS Clinical Trials Group [ACTG] CRS, Johannesburg, South Africa: Site 12301) CTU Grant 1U01AI069453-01; V Goncalves Veloso and M C da Silva Lourenco (Instituto de Pesquisa Clinica Evandro Chagas CRS, Rio de Janeiro, Brazil: Site 12101) CTU Grant 1U01 AI069476-01; A Mwafongo and A Moses (Malawi CRS, Lilongwe, Malawi: Site 12001) CTU Grant 1U01 AI069423-01; P Segura and C Ganoza (Barranco CRS, Lima, Peru: Site 11301; San Miguel CRS, Lima, Peru: Site 11302) CTU Grant 1U01 AI069438-01; G Kadzirange and R Mahachi (UZ Parirenyatwa CRS: Site 30313), CTU Grant 1U01 AI069436-01.

The project described was supported by Award Number UM1AI068636 from the National Institute of Allergy and Infectious Diseases, the National Institute of Dental and Cranio-facial Research (NIDCR), and UM1AI68634 for the Statistical and Data Management Center. The content is solely the responsibility of the authors and does not necessarily represent the official views of the National Institute of Allergy and Infectious Diseases or the National Institutes of Health.

\section{References}

1. Pichith K, Chanroeun H, Bunna P, et al. Clinical aspects of AIDS at the Calmette hospital in Phnom Penh, Kingdom of Cambodia A report on 356 patients hospitalized in the Medicine 'B' Department of the Calmette Hospital. Santé J. 2001; 11:17-23. [French].

2. Nissapatorn V, Lee CK, Rohela M, Anuar AK. Spectrum of opportunistic infections among HIVinfected patients in Malaysia. Southeast Asian J Trop Med Public Health. 2004; 35 (Suppl 2):S26S32.

3. Selwyn PA, Alcabes P, Hartel D, et al. Clinical manifestations and predictors of disease progression in drug users with human immunodeficiency virus infection. N Eng J Med. 1992; 327:1697-1703.

4. Katz MH, Greenspan D, Westenhouse J, et al. Progression to AIDS in HIV-infected homosexual and bisexual men with hairy leukoplakia and oral candidiasis. AIDS. 1992; 6:95-100. [PubMed: 1543572] 
5. Feigal DW, Katz MH, Greenspan D, et al. The prevalence of oral lesions in HIV-infected homosexual and bisexual men: three San Francisco epidemiological cohorts. AIDS. 1991; 5:519525. [PubMed: 1863403]

6. Melnick SL, Engel D, Truelove E, et al. Oral mucosal lesions: association with the presence of antibodies to the human immunodeficiency virus. Oral Surg Oral Med Oral Pathol. 1989; 68:37-43. [PubMed: 2755688]

7. Shiboski CH, Hilton JF, Greenspan D, et al. HIV-related oral manifestations in two cohorts of women in San Francisco. J Acquir Immune Defic Syndr. 1994; 7:964-971. [PubMed: 7914233]

8. Shiboski CH, Hilton JF, Neuhaus JM, Canchola A, Greenspan D. Human immunodeficiency virusrelated oral manifestations and gender. A longitudinal analysis. Arch Intern Med. 1996; 156:22492254. [PubMed: 8885825]

9. Arendorf TM, Bredekamp B, Cloete CA, Sauer G. Oral manifestations of HIV infection in 600 South African patients. J Oral Pathol Med. 1998; 27:176-179. [PubMed: 9563573]

10. Chidzonga MM. HIV/AIDS orofacial lesions in 156 Zimbabwean patients at referral oral and maxillofacial surgical clinics. Oral Dis. 2003; 9:317-322. [PubMed: 14629334]

11. Lamster IB, Begg MD, Mitchell-Lewis D, et al. Oral manifestations of HIV infection in homosexual men and intravenous drug users. Study design and relationship of epidemiologic, clinical, and immunologic parameters to oral lesions. Oral Surg Oral Med Oral Pathol. 1994; 78:163-174. [PubMed: 7936584]

12. Hodgson TA. HIV-associated oral lesions: prevalence in Zambia. Oral Dis. 1997; 3 (Suppl 1):S46S50. [PubMed: 9456656]

13. Schuman P, Ohmit SE, Sobel JD, et al. Oral lesions among women living with or at risk for HIV infection. HIV Epidemiology Research Study (HERS) Group. Am J Med. 1998; 104:559-564. [PubMed: 9674719]

14. Tukutuku K, Muyembe-Tamfum L, Kayembe K, Odio W, Kandi K, Ntumba M. Oral manifestations of AIDS in a heterosexual population in a Zaire hospital. J Oral Pathol Med. 1990; 19:232-234. [PubMed: 2359042]

15. Barr CE, Lopez MR, Rua-Dobles A, Miller LK, Mathur-Wagh U, Turgeon LR. HIV-associated oral lesions; immunologic, virologic and salivary parameters. J Oral Pathol Med. 1992; $21: 295-$ 298. [PubMed: 1355794]

16. Glick M, Muzyka BC, Lurie D, Salkin LM. Oral manifestations associated with HIV-related disease as markers for immune suppression and AIDS. Oral Surg Oral Med Oral Pathol. 1994; 77:344-349. [PubMed: 8015797]

17. Patton LL. Sensitivity, specificity, and positive predictive value of oral opportunistic infections in adults with HIV/AIDS as markers of immune suppression and viral burden. Oral Surg Oral Med Oral Pathol Oral Radiol Endod. 2000; 90:182-188. [PubMed: 10936837]

18. Shiboski CH, Neuhaus JM, Greenspan D, Greenspan JS. The effect of receptive oral sex and smoking on the incidence of hairy leukoplakia in HIV-positive gay men. J Acquir Immune Def Synd. 1999; 21:236-242.

19. Chidzonga MM, Mwale M, Malvin K, Martin JN, Greenspan JS, Shiboski CH. Oral candidiasis as a marker of HIV disease progression among Zimbabwean women. J Acquir Immune Defic Syndr. 2008; 47:579-584. [PubMed: 18176326]

20. Greenspan D, Komaroff E, Redford M, et al. Oral mucosal lesions and HIV viral load in the Women's Interagency HIV Study (WIHS). J Acquir Immune Defic Syndr. 2000; 25:44-50. [PubMed: 11064503]

21. Shiboski CH, Webster-Cyriaque JY, Ghannoum M, Greenspan JS, Dittmer D. Overview of the oral HIV/AIDS Research Alliance Program. Adv Dent Res. 2011; 23:28-33. [PubMed: 21441477]

22. Swindells S, Komarow L, Tripathy S, et al. Screening for pulmonary tuberculosis in HIV-infected individuals: AIDS Clinical Trials Group Protocol A5253. Int J Tuberc Lung Dis. 2013; 17:532539. [PubMed: 23485388]

23. World Health Organization. Global Health Atlas country profiles. Geneva, Switzerland: WHO; 2009. http://apps.who.int/globalatlas/predefinedreports/tb/index.asp [Accessed March 2009] 
24. Shiboski CH, Patton LL, Webster-Cyriaque JY, et al. The Oral HIV/AIDS Research Alliance: updated case definitions of oral disease endpoints. J Oral Pathol Med. 2009; 38:481-488. [PubMed: 19594839]

25. World Health Organization. WHO/HTM/TB/2012.6. Geneva, Switzerland: WHO; 2012. Global tuberculosis report, 2012. http://www.who.int/tb/publications/global_report/en/ [Accessed March 2014]

26. World Health Organization. WHO/HTM/TB/2012.1. WHO/HIV/2012.1. Geneva, Switzerland: WHO; 2012. WHO policy on collaborative TB/HIV activities: guidelines for national programmes and other stakeholders. http://www.who.int/tb/publications/2012/

tb_hiv_policy_9789241503006/en/index.html [Accessed March 2014]

27. Holmes CB, Wood R, Badri M, et al. CD4 decline and incidence of opportunistic infections in Cape Town, South Africa: implications for prophylaxis and treatment. J Acquir Immune Defic Syndr. 2006; 42:464-469. [PubMed: 16810113]

28. Lawn SD, Dheda K, Kerkhoff AD, et al. Determine TB-LAM lateral flow urine antigen assay for HIV-associated tuberculosis: recommendations on the design and reporting of clinical studies. BMC Infect Dis. 2013; 13:407. [PubMed: 24004840] 

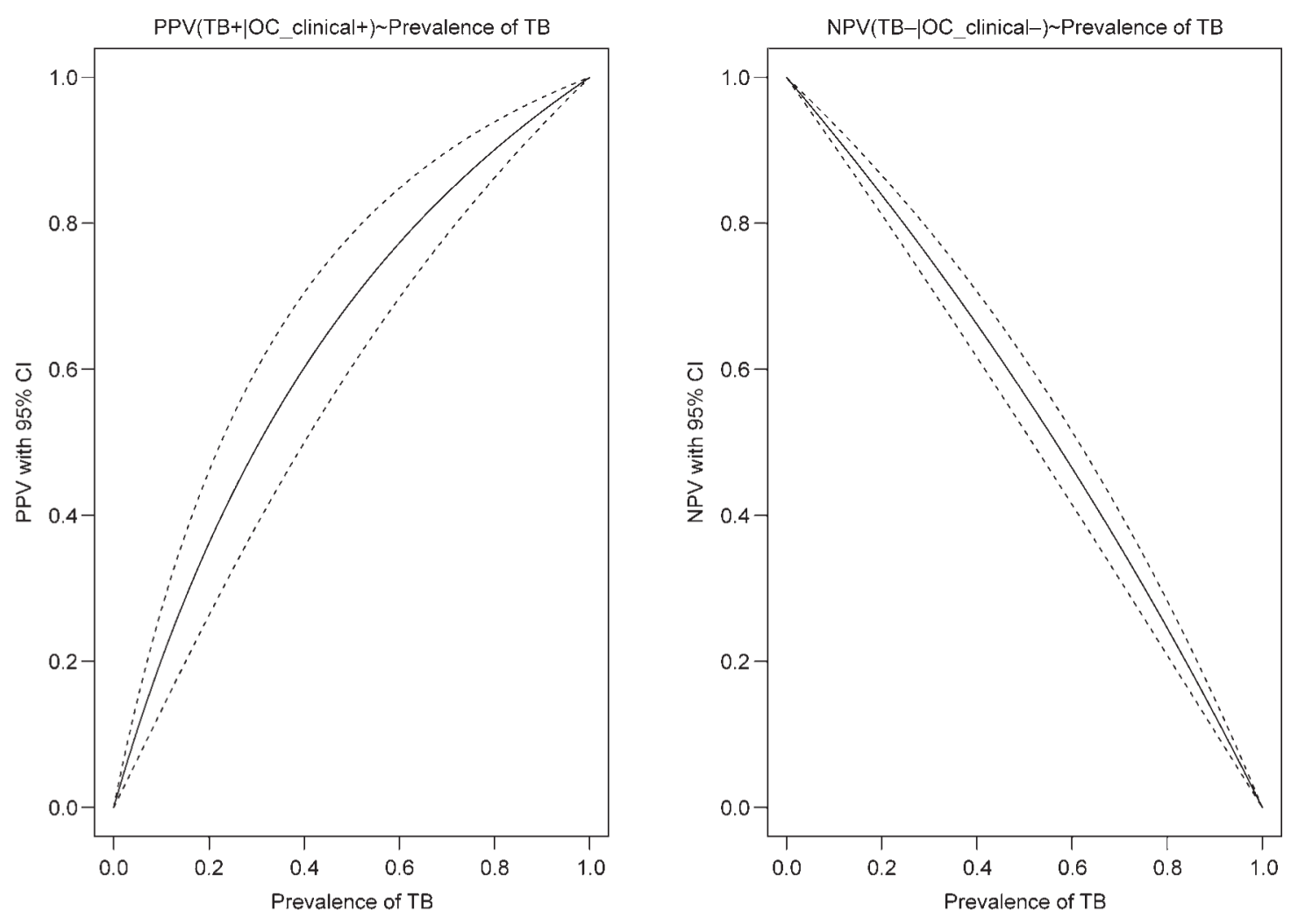

Figure.

PPVs and NPVs of clinical diagnosis of oral candidiasis in predicting TB in the Africa sites participating in Protocol A5253. PPV = positive predictive value; $\mathrm{OC}=$ oral candidiasis; $\mathrm{TB}$ $=$ tuberculosis NPV $=$ negative predictive value $; \mathrm{CI}=$ confidence interval . 


\section{Table 1}

Baseline characteristics among 454 participants with an interpretable sputum culture in Protocol A5253 (subSaharan Africa sites) ${ }^{*}$

\begin{tabular}{|c|c|}
\hline Characteristic & $n(\%)$ \\
\hline \multicolumn{2}{|l|}{ Categorical variables } \\
\hline \multicolumn{2}{|l|}{ Race/ethnicity } \\
\hline Black Non-Hispanic & $451(99)$ \\
\hline Hispanic (any race) & $2(<1)$ \\
\hline Asian/Pacific Islander & $1(<1)$ \\
\hline \multicolumn{2}{|l|}{ Sex } \\
\hline Female & $321(71)$ \\
\hline Male & $133(29)$ \\
\hline Sputum culture-positive for tuberculosis & $54(12)$ \\
\hline \multicolumn{2}{|l|}{ Oral candidiasis } \\
\hline Clinical diagnosis & $81(18)$ \\
\hline Culture-confirmed diagnosis $^{\dagger}$ & $62(14)$ \\
\hline Currently on antifungal medication & $36(8)$ \\
\hline \multicolumn{2}{|l|}{ Continuous variables, median (range) } \\
\hline Age & $33(18-65)$ \\
\hline CD4 cell count & $257(1-1796)$ \\
\hline
\end{tabular}


Table 2

Prevalence of oral candidiasis and other oral mucosal lesions by TB status (as determined using sputum culture) among 454 participants in Protocol A5253

\begin{tabular}{|c|c|c|c|}
\hline Oral lesion (clinical diagnosis) & $\begin{array}{c}\text { TB-positive }(n=54) \\
n(\%)\end{array}$ & $\begin{array}{c}\text { TB-negative }(n=400) \\
n(\%)\end{array}$ & $P$ value \\
\hline \multicolumn{4}{|l|}{ Candidiasis } \\
\hline Any oral candidiasis & $19(35)$ & $62(16)$ & $<0.001^{*}$ \\
\hline Pseudomembranous candidiasis & $18(33)$ & $48(12)$ & $<0.001^{*}$ \\
\hline Erythematous candidiasis & $2(4)$ & $16(4)$ & $1.00^{\dagger}$ \\
\hline Angular cheilitis & $0(0)$ & $5(1)$ & $1.00^{\dagger}$ \\
\hline \multicolumn{4}{|l|}{ Other oral mucosal lesions } \\
\hline Hairy leukoplakia & $1(2)$ & $6(2)$ & $0.59^{\dagger}$ \\
\hline Necrotizing ulcerative gingivitis/periodontitis & $2(4)$ & $4(1)$ & $0.15^{\dagger}$ \\
\hline Recurrent oral/labial herpes simplex infection & $2(4)$ & $4(1)$ & $0.15^{\dagger}$ \\
\hline Kaposi's sarcoma & $0(0)$ & $3(<1)$ & $1.00^{\dagger}$ \\
\hline Oral warts & $0(0)$ & $2(<1)$ & $1.00^{\dagger}$ \\
\hline Ulceration not otherwise specified & $0(0)$ & $1(<1)$ & $1.00^{\dagger}$ \\
\hline \multicolumn{4}{|l|}{$\chi^{2}$ test. } \\
\hline 'Fisher's exact test. & & & \\
\hline
\end{tabular}




\section{Table 3}

Baseline characteristics by diagnosis of oral candidiasis among 454 participants with an interpretable sputum culture in Protocol A5253 (sub-Saharan Africa sites)*

\begin{tabular}{|c|c|c|c|}
\hline Characteristic & $\begin{array}{c}\text { Oral candidiasis }(n=81) \\
n(\%)\end{array}$ & $\begin{array}{l}\text { No oral candidiasis }(n=373) \\
n(\%)\end{array}$ & $P$ value $^{\dagger}$ \\
\hline \multicolumn{4}{|l|}{ Categorical variables } \\
\hline \multicolumn{4}{|l|}{ Race/ethnicity } \\
\hline Black non-Hispanic & $81(100)$ & $370(99)$ & 1.000 \\
\hline Hispanic (any race) & $0(0)$ & $2(1)$ & \\
\hline Asian/Pacific Islander & $0(0)$ & $1(<1)$ & \\
\hline \multicolumn{4}{|l|}{ Sex } \\
\hline Female & $53(65)$ & $268(72)$ & 0.250 \\
\hline Male & $28(35)$ & $105(28)$ & \\
\hline \multicolumn{4}{|l|}{ CD4 cell count, cells $/ \mathrm{mm}^{3}$} \\
\hline$<200$ & $55(68)$ & $96(26)$ & $<0.001$ \\
\hline $200-500$ & $22(27)$ & $202(54)$ & \\
\hline$>500$ & $4(5)$ & $75(20)$ & \\
\hline Currently on antifungal medication & $8(10)$ & $28(8)$ & 0.496 \\
\hline \multicolumn{4}{|l|}{ Continuous variables, median $(\mathrm{Q} 1, \mathrm{Q} 3)^{*}$} \\
\hline Age & $35(29,40)$ & $32(28,39)$ & 0.108 \\
\hline CD4 cell count & $151(46,228)$ & $287(190,463)$ & $<0.001$ \\
\hline
\end{tabular}




\section{Table 4}

Multivariate model exploring the association between tuberculosis (outcome) and oral candidiasis controlling for CD4 cell count, and antifungal medication among 454 Protocol A5253 participants recruited from African sites

\begin{tabular}{lcc}
\hline Independent variables & aOR $(\mathbf{9 5 \%}$ CI) & $\boldsymbol{P}$ value \\
\hline Clinical diagnosis of oral candidiasis (vs. no oral candidiasis) & $2.38(1.22-4.66)$ & 0.01 \\
CD4 cell count, cells $/ \mathrm{mm}^{3}$ (per 50 unit increase) & $0.92(0.84-0.99)$ & 0.04 \\
Antifungal medication (yes vs. no) & $0.16(0.02-1.19)$ & 0.07 \\
\hline
\end{tabular}

$\mathrm{aOR}=$ adjusted odds ratio $\mathrm{CI}=$ confidence interval. 